\title{
A Convergent three Field Quadrilateral Finite Element Method for Simulating Viscoelastic Flow on Irregular Meshes
}

\author{
Victoriano Ruas
}

Pontificia Universidade do Rio de Janeiro (PUC/RJ)

Brazil (currently on leave)

Universite de Saint-Etienne \&

LMM, URA 229 CNRS, tour 66, 4e étage

75252 Paris, cedex 05

ABSTRACT. In a recent paper a finite element method for solving the three field Stokes system as a basis for the numerical solution of viscoelastic fluid flow problems was introduced. The method constructed upon a biquadratic velocity, a discontinuous linear pressure and a bilinear extra stress tensor interpolation in quadrilaterals, enriched with fifteen bubble tensors, has been proved to yield second order approximations of these variables, in the case of rectangular meshes. In this work equivalent results are proven to hold in the case of irregular meshes.

RÉSUMÉ. Dans un article récent a été introduite une méthode d'éléments finis pour la résolution du système de Stokes à trois champs en tant que base pour la simulation numérique d'écoulements de fluides viscoélastiques. Cette méthode, basée sur les interpolations biquadratiques pour la vitesse, linéaire discontinue pour la pression et bilinéaire enrichie avec quinze tenseurs bulle pour le tenseur d'extracontraintes sur des quadrangles, fournit des approximations du second ordre de ces trois champs, dans le cas d'éléments rectangulaires. Dans cet article, on démontre des résultats analogues dans le cas de maillages irréguliers.

KEY WORDS : éléments finis, fluides visco-élastiques, maillages irréguliers, quadrangles, systèmes de Stokes, trois champs.

MOTS-CLÉs : finite elements, irregular mesh, quadrilateral, Stokes system, three field, viscoelastic flow.

Revue européenne des éléments finis. Volume 1 - n 4/1992, pages 391 à 406 


\section{Introduction}

An accurate numerical solution of the three field Stokes system is generally acknowledged as a basic requirement to be fulfilled by any candidate finite element method for a successful simulation of viscoelastic flows. Let us recall that in the case where the velocity vanishes on the boundary of the flow domain $\Omega$ of $\mathcal{R}^{2}$ assumed here to be a polygon, such a system in variational form may be expressed as problem (1), namely:

$$
\begin{aligned}
& \text { Find } \sigma \in\left[L^{2}(\Omega)\right]_{\text {aym }}^{2 x^{2}}, u \in\left[H_{0}^{1}(\Omega)\right. \\
& -(\sigma, \mathcal{E}(v))+(p, \operatorname{div} v)=(f, v) \\
& \frac{1}{2 \eta}(\sigma, \tau)-(\tau, \mathcal{E}(u))=0 \\
& (q, \operatorname{div} u)=0
\end{aligned}
$$

such that

$$
\forall v \in\left[H_{0}^{1}(\Omega)\right]^{2}
$$$$
\forall \tau \in\left[L^{2}(\Omega)\right]_{\Delta y m}^{2 x 2}
$$$$
\forall q \in L^{2}(\Omega) / \mathcal{R}
$$

where $(.,$.$) represents the standard inner product of L^{2}(\Omega), f$ is a field of given body forces, $\eta$ is the viscosity of the fluid and $u, \sigma$, p represent the velocity, extra stress tensor and pressure fields respectively. As usual $\mathcal{E}(v)$ denotes the symmetric gradient of a vector field $v$.

In a recent work, SANDRI [SAN 91] proved that, at least for viscoelastic fluids of the Oldroyd B - type, second order finite element methods for system (1) do produce convergent approximations, though of order $3 / 2$. At the present state of the art, it may be legitimately conjectured that a similar conclusion applies to Maxwell fluids as well, although in this case some compatibility condition between the approximation spaces for $u$ and $\sigma$ such as ( 3 ) given hereafter, must be necessarily satisfied ( see e. g. [RUA 85]).

In [RUA 91] and [RUA 93] two optimal three-field quadrilateral finite element methods to solve system (1), with a continuous extra stress representation were proposed and studied. Notice that, while such a continuity property is generally required in viscoelastic flow simulations, some degrees of freedom must be attached to the interior of the elements in order to satisfy the above mentioned condition. However, in those works the methods were only proved to converge in the case of rectangular meshes. In this paper the author proves that the element introduced in [RUA 93] is second order convergent in the natural norms for system (1), in the case of irregular meshes too. As pointed out above, one may expect that SANDRI's results [SAN 91] for Oldroyd - B fluids do apply as well to this new element, even in the case of a Maxwell fluid. 


\section{The New Finite Element Method}

Let $\left\{\mathcal{T}_{h}\right\}_{h}$ be a family of partitions of $\Omega$ into non degenerated convex quadrilaterals, satisfying the usual quasiuniformity conditions. For the later convenience, we define $\mathrm{h}=\max _{K \in \tau_{h}} h_{K}$, where $h_{K}$ is the maximum edge length of quadrilateral $\mathrm{K} \in \mathcal{T}_{h}$. We recall below the natural approximate problem for system (1), associated with every $\mathcal{T}_{h}$ :

$$
\left.\begin{array}{ll}
\text { Find }\left(\sigma_{h}, u_{h}, p_{h}\right) \in T_{h} \times V_{h} \times Q_{h}, & \text { such that } \\
-\left(\sigma_{h}, \mathcal{E}(v)\right)+\left(p_{h}, \operatorname{div} v\right)=(f, v) & \forall v \in V_{h} \\
\frac{1}{2 \eta}\left(\sigma_{h}, \tau\right)-\left(\tau, \mathcal{E}\left(u_{h}\right)\right)=0 & \forall \tau \in T_{h} \\
\left(q, \operatorname{div} u_{h}\right)=0 & \forall q \in Q_{h}
\end{array}\right\}
$$

where $(.,$.$) ) denotes the standard L^{2}(\Omega)$ inner product, and $Q_{h}=\tilde{Q}_{h} / \mathcal{R}$, $V_{h}$ and $\tilde{Q}_{h}$ being defined as in two well-known mixed methods for solving the Stokes system in terms of velocity and pressure (see e.g. FORTIN \& FORTIN [FOR 89] and BERCOVIER \& PIRONNEAU [BER 79] respectively ), namely:

- $V_{h}$ : In both cases the velocity space is based on standard Lagrangian isoparametric biquadratic functions for quadrilaterals with straight edges.

- $\bar{Q}_{h}$ is either the space of (discontinuous) functions which are linear over each quadrilateral of $\mathcal{T}_{h}$, or the space of continuous piecewise isoparametric bilinear functions, associated with $T_{h}$.

Like in [RUA 93] space $T_{h}$ is defined as the direct sum of two spaces $T_{h}^{1}$ and $T_{h}^{2}$. Here $T_{h}^{1}$ is the space of standard Lagrangian isoparametric bilinear symmetric tensors associated with $\mathcal{T}_{h} . T_{h}^{2}$ in turn is the space of tensors that vanish on the boundary of the elements of $\mathcal{T}_{h}$, spanned by fifteen tensors to be specified later on. For the moment, let us just recall that according to [FOR 88] and [RUA X], the following compatibility condition, leads to optimal second order convergence results for problem (2), with the above definition of $V_{h}, T_{h}^{1}$ and $Q_{h}$ :

There exists a strictly positive constant $\beta$ independent of $h$ such that:

$$
\inf _{v \in V_{h}-\{0\}} \quad \sup _{T} T_{h}-\{0\} \quad \frac{(\tau, \mathcal{E}(v))}{\|\tau\|_{0} \mid v \|_{1}} \geq \beta
$$

where $\|\cdot\|_{0}=(., .)^{\frac{1}{2}}$ and $|v|_{1}=\|\operatorname{grad} v\|_{0}$. 
According to [FOR 88] and [RUA X], the key steps to establish condition (3) may be described in the following manner:

Consider an arbitrary quadrilateral $K$ of $\mathcal{T}_{h}$. Let $\hat{K}$ be the unity reference square $(-1,+1) \times(-1,1)$ in the cartesian plane $(\hat{O}, \vec{x}, \vec{y})$ and $\mathcal{F}_{K}$ be the invertible bilinear mapping from $\hat{K}$ onto $K$.

Let now $\left\{\widehat{\mathcal{E}}_{i}^{K}\right\}_{i=1}^{M}$ be a set of linearly independent tensors defined in $\widehat{K}$, such that the restriction to $\mathrm{K}$ of $\mathcal{E}(v)$ for any $v \in V_{h}$ may be expressed by

$$
\mathcal{E}(v)_{/ K} \circ \mathcal{F}_{K}=\frac{h_{K}}{J_{K}} \sum_{i=1}^{M} \alpha_{i} \widehat{\mathcal{E}}_{i}^{K}
$$

where $\vec{\alpha}=\left\{\alpha_{i}\right\}_{i=1}^{M}$ is a vector of real coefficients and $J_{K}$ is the jacobian of $\mathcal{F}_{K}$. Assume that the $L^{2}(\widehat{K})$-norm of $\widehat{\mathcal{E}}_{i}^{K}$ is bounded above independently of $h_{K}$. Now define $M$ linearly independent tensors $\left\{\sigma_{j}\right\}_{i=1}^{M}$ independent of $h$ that vanish on the boundary of $\widehat{K}$, such that the $\mathrm{M} \times \mathrm{M}$ matrix $A=\left\{a_{i j}\right\}$,

$$
a_{i j}=\int_{\widehat{K}} \widehat{\mathcal{E}}_{i}^{K} \cdot \sigma_{j} d x d y
$$

is invertible. Here the dot denotes the standard inner product of $\mathcal{R}^{\prime}, 1 \geq 1$.

In this way we shall define space $T_{h}^{2}$ to be the one of tensors whose restriction to every $\mathrm{K} \in \mathcal{T}_{h}^{2}$ belongs to the space spanned by $\left\{\sigma_{j}^{K}\right\}_{j=1}^{M}$, where $\sigma_{j}^{K}=\sigma_{j} \circ \mathcal{F}_{K}^{-1}$. In so doing $\forall \sigma \in T_{h}$ we have :

$$
\sigma_{/ K}=\sum_{j=1}^{M} \gamma_{j} \sigma_{j}^{K} \quad \forall K \in \mathcal{T}_{h}
$$

where $\vec{\gamma}=\left\{\gamma_{i}\right\}_{i=1}^{M}$ is a vector of real coefficients, and

$$
(\sigma, \mathcal{E}(v))=\sum_{K \in T_{h}} \sum_{j=1}^{M} \gamma_{j} \int_{K} \sigma_{j}^{K} \cdot \mathcal{E}(v) d K=\sum_{K \in \mathcal{T}_{h}} h_{K} A \vec{\gamma} \cdot \vec{\alpha}
$$

According to the arguments developped in [RUA X], since all the coefficients of matrix $A$ are bounded above by constants independent of $h$ by assumption, the fact that $|\operatorname{det} A|$ is bounded below by a non zero constant independent of $h$ is all that is needed to establish (3). In fact this is a consequence of the fact that in this case $\left\|A^{-1}\right\|$ is bounded above independently of $h$.

Actually among several different possibilities, the space $T_{h}^{2}$ selected in this work, is the same as the one used in [RUA 93] for the case of rectangles. More specifically this space is locally spanned by fifteen symmetric tensors $\left\{\sigma_{j}^{K}\right\}_{j=1}^{15}$ associated through mapping $\mathcal{F}_{K}$ with the tensors $\left\{\sigma_{j}\right\}_{j=1}^{15}$ defined in $\widehat{K}$ as follows :

First set $\Psi(x, y)=\left(1-x^{2}\right)\left(1-y^{2}\right)$, and next 


$$
\sigma_{j}=\Psi \widehat{\sigma}_{j} \text { for } j=\overline{1,15}
$$

where the $\widehat{\sigma}_{j}^{\prime}$ referred to the frame $(O, \vec{s}, \vec{t})$ to be defined in Section 3 , are represented by:

$$
\begin{array}{lll}
\widehat{\sigma}_{1}=\left(\begin{array}{ll}
1 & 0 \\
0 & 0
\end{array}\right) ; & \widehat{\sigma}_{2}=\left(\begin{array}{cc}
0 & 0 \\
0 & 1
\end{array}\right) ; & \widehat{\sigma}_{3}=\left(\begin{array}{ll}
0 & 1 \\
1 & 0
\end{array}\right) ; \\
\widehat{\sigma}_{4}=\left(\begin{array}{ll}
x & 0 \\
0 & 0
\end{array}\right) ; & \widehat{\sigma}_{5}=\left(\begin{array}{cc}
0 & 0 \\
0 & x
\end{array}\right) ; & \widehat{\sigma}_{6}=\left(\begin{array}{cc}
0 & x \\
x & 0
\end{array}\right) ; \\
\widehat{\sigma}_{7}=\left(\begin{array}{ll}
y & 0 \\
0 & 0
\end{array}\right) ; & \widehat{\sigma}_{8}=\left(\begin{array}{cc}
0 & 0 \\
0 & y
\end{array}\right) ; & \widehat{\sigma}_{9}=\left(\begin{array}{cc}
0 & y \\
y & 0
\end{array}\right) ; \\
\widehat{\sigma}_{10}=\left(\begin{array}{ll}
x y & 0 \\
0 & 0
\end{array}\right) ; & \widehat{\sigma}_{11}=\left(\begin{array}{cc}
y^{2}-x^{2} & 0 \\
0 & 0
\end{array}\right) ; & \widehat{\sigma}_{12}=\left(\begin{array}{ll}
0 & x^{2} y \\
x^{2} y & 0
\end{array}\right) ; \\
\widehat{\sigma}_{13}=\left(\begin{array}{ll}
0 & 0 \\
0 & x y
\end{array}\right) ; & \widehat{\sigma}_{14}=\left(\begin{array}{cc}
0 & 0 \\
0 & x^{2}-y^{2}
\end{array}\right) ; & \widehat{\sigma}_{15}=\left(\begin{array}{ll}
0 & x y^{2} \\
x y^{2} & 0
\end{array}\right) .
\end{array}
$$

As shown hereafter, the so-defined space $T_{h}^{2}$ is such that not only matrix $A$ defined by (5) is invertible but $\operatorname{det} A$ is bounded below by a non-zero constant independent of $h$. According to the arguments above the associated space $T_{h}$ satisfies condition (3) as required.

\section{Convergence Results}

Let us first recall the following results for rectangles given in [CAR 91], [RUA 93] and [RUA 92].

Assume that $\mathcal{T}_{h}$ consists of rectangles whose edges are parallel to the cartesian axes $O \vec{x}$ and $O \vec{y}$. If $\mathrm{K} \in \mathcal{T}_{h}$ we may write $\mathcal{F}_{K}=\left\{\frac{l_{x} x}{2}+x_{0}, \frac{l_{y} y}{2}+y_{0}\right\}$ where $x_{0}$ and $y_{0}$ are constants, and $l_{x}$ and $l_{y}$ are the lengths of the edges of $K$. Setting $r_{K}=\frac{l_{y}}{l_{x}}$, from the initial assumption on $\left\{\mathcal{T}_{h}\right\}_{h}$, we may assert that there exists two constants $\xi$ and $\rho$ independent of $h$, such that $r_{K} \geq \rho$ and $r_{K}^{-1} \geq \xi \quad \forall K \in \mathcal{T}_{h}$.

One may choose the $\hat{\mathcal{E}}_{i}^{K}$ 's referred to the frame $(O, \vec{x}, \vec{y})$ to be the fifteen tensors given by: 


$$
\begin{array}{ll}
\widehat{\mathcal{E}}_{i}^{K}=\widehat{\sigma}_{i} & \text { for } i=\overline{1,9} \\
\widehat{\mathcal{E}}_{10}^{K}=\left(\begin{array}{ll}
x y & x^{2} / 4 r_{K} \\
x^{2} / 4 r_{K} & 0
\end{array}\right) ; & \widehat{\mathcal{E}}_{11}^{K}=\left(\begin{array}{ll}
y^{2} & x y / r_{K} \\
x y / r_{K} & 0
\end{array}\right) ; \\
\widehat{\mathcal{E}}_{12}^{K}=\left(\begin{array}{ll}
2 r_{K} x y^{2} & x^{2} y \\
x^{2} y & 0
\end{array}\right) ; & \widehat{\mathcal{E}}_{13}^{K}=\left(\begin{array}{ll}
0 & r_{K} y^{2} / 4 \\
r_{K} y^{2} / 4 & x y
\end{array}\right) ; \\
\widehat{\mathcal{E}}_{14}^{K}=\left(\begin{array}{ll}
0 & r_{K} x y \\
r_{K} x y & x^{2}
\end{array}\right) ; & \widehat{\mathcal{E}}_{15}^{K}=\left(\begin{array}{ll}
0 & x y^{2} \\
x y^{2} & 2 x^{2} y / r_{K}
\end{array}\right)
\end{array}
$$

In so doing, the determinant of matrix $A$ is found to be strictly positive and approximately equal to $0.1282152754 \times 10^{-7}, \forall \mathrm{K} \in \mathcal{T}_{h}$, which implies that property (3) holds, as recalled in Section 2.

Let us now go back to the case where $K$ is an arbitrary quadrilateral of $\mathcal{T}_{h}$. First of all observe that $K$ can be viewed as the deformation of a rectangle with edge lengths equal to $l_{x}$ and $l_{y}$, parametrized by three angular increments $\theta, \phi_{1}$ and $\phi_{2}$ in the following manner.

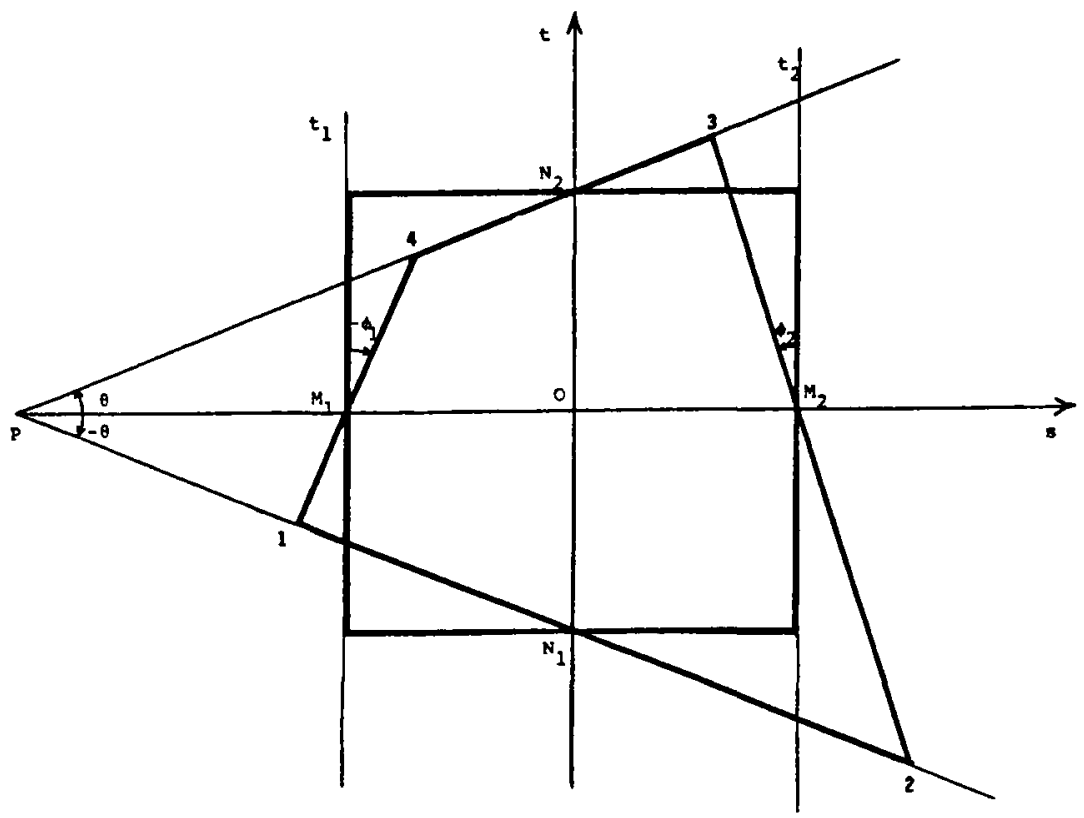

A convex quadrilateral regarded as the deformation of a rectangle Figure 1

Let $\theta$ and $\theta$ be the acute half angles between the two pairs of opposite edges of $K$ (refer to figure 1). Take $\theta$ such that $0 \leq \theta \leq \theta^{\prime}$ and consider the pair of 
opposite edges of $K$ corresponding to this angle if $\theta \neq \theta^{\circ}$. If $\theta=\theta^{*}$ choose this pair arbitrarily.

If $\theta \neq 0$ take the intersection $P$ of the prolongations of these edges of $K$ and let $s$ be the bissector of the underlying corner with vertex in P. Let also $M_{1}$ and $M_{2}$ be the intersections of $s$ with the two other edges, and $t_{1}$ and $t_{2}$ be the perpendiculars to $s$ passing through $M_{1}$ and $M_{2}$ respectively. Finally let $t$ be the parallel to $t_{1}$ and $t_{2}$ passing through the mid-point $O$ of the segment $M_{1} M_{2}$, and $N_{1}$ and $N_{2}$ be the intersections of $t$ with the two edges of $\mathrm{K}$ that do not contain $M_{1}$ and $M_{2}$. In this case $\mathrm{R}$ is the rectangle whose edges are parallel to $s$ and $t$, and whose edge mid-points are $M_{1}, M_{2}, N_{1}$ and $N_{2}$.

If $\theta=0$, construct $\mathrm{R}$ in the same manner, starting from line $s$, parallel to the two parallel edges and passing through the mid-points $M_{1}$ and $M_{2}$ of the two other edges of $K$.

Now we define $\phi_{i}$ to be the angle formed by the edge of $K$ and the edge of $R$ that pass through $M_{i}$ for $\mathrm{i}=1,2$, measured from the latter towards the former, and both oriented in the same given sense, in such a way that $-\pi / 2 \leq \phi_{i} \leq \pi / 2$. In so doing the so-called deformation of $\mathrm{R}$ onto $\mathrm{K}$ is completely defined.

Here again we set $l_{x}=$ length $\left(M_{1} M_{2}\right), l_{y}=\operatorname{length}\left(N_{1} N_{2}\right)$, and $r_{K}=l_{y} / l_{x}$. In so doing, one may easily establish that $0 \leq \operatorname{tg} \theta<r_{K}$, whereas $\theta^{\prime}=\left|\phi_{1}-\phi_{2}\right| / 2$. Now refer the mapping $\mathcal{F}_{K}$ to the direct cartesian coordinate system $(O, \vec{s}, \vec{t})$, where $\vec{s}$ and $\vec{t}$ are the unit vectors of $s$ and $t$ respectively, in such a way that $\vec{s}$ is oriented from $P$ to $O$ if $\theta \neq 0$, or in an arbitrarily chosen way otherwise. In so doing we assume that $\phi_{1}$ and $\phi_{2}$ are positive in the trigonometric sense.

Now set $\alpha=\operatorname{tg} \theta, \beta_{1}=\operatorname{tg} \phi_{1}, \beta_{2}=\operatorname{tg} \phi_{2}$, and $\delta_{K}=\max \left(\alpha,\left|\beta_{1}\right|,\left|\beta_{2}\right|\right)$, and define

$$
\delta=\sup _{h} \max _{K \in \mathcal{T}_{h}} \delta_{K}
$$

Notice that $\delta_{K}$ is a measure of the distortion of a quadrilateral $\mathrm{K}$ with respect to a rectangle.

Now we refer again to Figure 1 , where the vertices of quadrilateral $\mathrm{K}$ are numbered from one to four as indicated. The values of the coordinates $\left(x_{i}, y_{i}\right)$ of the $\mathrm{i}$-th vertex in system $s O t, \mathrm{i}=1,2,3,4$ are given respectively by

$$
\begin{aligned}
& \mathbf{x}_{1}=\frac{1_{x}}{2} \frac{\mathrm{r}_{K} \beta_{1}-1}{1+\alpha \beta_{1}} \quad \mathbf{y}_{1}=\frac{l_{x}}{2} \frac{\alpha-\mathrm{r}_{K}}{1+\alpha \beta_{1}} \\
& \mathbf{x}_{2}=\frac{\mathrm{l}_{\mathrm{x}}}{2} \frac{1-\mathrm{r}_{\mathrm{K}} \beta_{2}}{1+\alpha \beta_{2}} \quad \mathbf{y}_{2}=\frac{\mathrm{l}_{\mathrm{x}}}{2} \frac{-\mathrm{r}_{K}-\alpha}{1+\alpha \beta_{2}} \\
& x_{3}=\frac{l_{x}}{2} \frac{1+r_{K} \beta_{2}}{1-\alpha \beta_{2}} \quad y_{3}=\frac{l_{x}}{2} \frac{r_{K}+\alpha}{1-\alpha \beta_{2}} \\
& \mathbf{x}_{4}=\frac{l_{x}}{2} \frac{-1+r_{K} \beta_{1}}{1-\alpha \beta_{1}} \quad y_{4}=\frac{l_{x}}{2} \frac{r_{k}-\alpha}{1-\alpha \beta_{1}}
\end{aligned}
$$


In so doing, we may express mapping $\mathcal{F}_{K}$ in terms of the $\left(x_{i}, y_{i}\right)$ 's in the following manner. Let $(s, t)=\mathcal{F}_{K}(x, y)$, where $x$ and $y$ are the coordinates of a point in the reference plane $x \hat{O} y$. We have

$$
\begin{aligned}
& s=\bar{x}+f x+d y-e x y \\
& t=\bar{y}+c x+a y-b x y
\end{aligned}
$$

where $4 \bar{x}=\sum_{i=1}^{4} x_{i}$ and $4 \bar{y}=\sum_{i=1}^{4} y_{i}$, and

$$
\begin{array}{ll}
4 a=y_{4}-y_{1}-y_{2}+y_{3} & 4 d=x_{4}-x_{1}-x_{2}+x_{3} \\
4 b=y_{2}-y_{1}+y_{4}-y_{3} & 4 e=x_{2}-x_{1}+x_{4}-x_{3} \\
4 c=y_{2}-y_{1}-y_{4}+y_{3} & 4 f=x_{2}-x_{1}-x_{4}+x_{3}
\end{array}
$$

Actually, using (9), and setting $D=4\left(1-\alpha^{2} \beta_{1}^{2}\right)\left(1-\alpha^{2} \beta_{2}^{2}\right) / l_{x}$ the values of $a, b, c, d, e, f$ in terms of $r_{K}, \alpha, \beta_{1}$ and $\beta_{2}$, are found to be respectively:

$$
\begin{aligned}
& a=\left[r_{K}\left(2-\alpha^{2} \beta_{1}^{2}-\alpha^{2} \beta_{2}^{2}\right)+\alpha^{3}\left(\beta_{1}^{2}+\beta_{2}^{2}\right)\right] / D \\
& b=\left[\alpha\left(-2+\alpha^{2} \beta_{1}^{2}+\alpha^{2} \beta_{2}^{2}\right)+r_{K} \alpha^{2}\left(\beta_{1}^{2}-\beta_{2}^{2}\right)\right] / D \\
& c=\left[\left(1-\alpha^{2} \beta_{1} \beta_{2}\right)\left[\left(\beta_{2}-\beta_{1}\right) r_{K} \alpha+\left(\beta_{1}+\beta_{2}\right) \alpha_{2}\right]\right] / D \\
& d=\left[\beta_{1}\left(r_{K}-\alpha\right)\left(1-\alpha^{2} \beta_{2}^{2}\right)+\beta_{2}\left(r_{K}+\alpha\right)\left(1-\alpha^{2} \beta_{1}^{2}\right)\right] / D \\
& e=\left[\beta_{2}\left(r_{K}+\alpha\right)\left(1-\alpha^{2} \beta_{1}^{2}\right)+\beta_{1}\left(r_{K}-\alpha\right)\left(1-\alpha^{2} \beta_{2}^{2}\right)\right] / D \\
& f=\left[2-\alpha^{2}\left(\beta_{1}^{2}+\beta_{2}^{2}\right)+r_{K} \alpha\left(\beta_{2}^{2}-\beta_{1}^{2}\right)\right] / D
\end{aligned}
$$

Moreover, by simply observing Figure 1, one concludes that both $a$ and $f$ are strictly positive.

Taking the above remarks and expressions as a starting point we first establish

Lemma 1 For every $v \in V_{h}$ and $\forall K \in \mathcal{T}_{h}$ we have in the local bases $(\vec{s}, \vec{t})$

$$
\mathcal{E}(v)_{\mid K} \circ \mathcal{F}_{K}=\frac{h_{K}}{J_{K}} \sum_{i=1}^{16} \beta_{i} \overline{\mathcal{E}}_{i}^{K}
$$

where $\left\{\beta_{i}\right\}_{i=1}^{16}$ is a convenient set of real coefficients associated with $v$ and tensors $\tilde{\mathcal{E}}_{i}^{K}$ in the system $s \mathrm{Ot}$ are represented by: 


$$
\begin{aligned}
& \tilde{\mathcal{E}}_{1}^{K}=\left[\begin{array}{ll}
1-\frac{b x}{a} & -\frac{d}{2 a}+\frac{e}{2 a} x \\
-\frac{d}{2 a}+\frac{e}{2 a} x & 0
\end{array}\right] \\
& \overline{\mathcal{E}}_{2}^{K}=\left[\begin{array}{ll}
0 & \frac{1}{2}-\frac{b}{2 a} x \\
\frac{1}{2}-\frac{b}{2 a} x & -\frac{d}{a}+\frac{c}{a} x
\end{array}\right] \\
& \dot{\mathcal{E}}_{3}^{K}=\left[\begin{array}{ll}
-\frac{c}{f}+\frac{b}{f} y & \frac{1}{2}-\frac{e}{2 f} y \\
\frac{1}{\tilde{2}}-\frac{e}{2 f} y & 0
\end{array}\right] \\
& \dot{\mathcal{E}}_{4}^{K}=\left[\begin{array}{ll}
0 & -\frac{c}{2 f}+\frac{b}{2 f} y \\
-\frac{c}{2 f}+\frac{b}{2 f} y & 1-\frac{e}{f} y
\end{array}\right] \\
& \tilde{\mathcal{E}}_{5}^{K}=\left[\begin{array}{ll}
x-\frac{b}{a} x^{2} & -\frac{d}{2 a} x+\frac{e}{2 a} x^{2} \\
-\frac{d}{2 a} x+\frac{e}{2 a} x^{2} & 0
\end{array}\right] \\
& \dot{\mathcal{E}}_{6}^{K}=\left[\begin{array}{ll}
\frac{a c}{f^{2}} y-\frac{a b}{f^{2}} y^{2} & -\frac{c}{2 f} x+\frac{a e}{2 f^{2}} y^{2} \\
-\frac{c}{2 f} x+\frac{a e}{2 f^{2}} y^{2} & x-\frac{d}{f} y
\end{array}\right] \\
& \tilde{\mathcal{E}}_{\gamma}^{K}=\left[\begin{array}{ll}
0 & x-\frac{b}{a} x^{2} \\
x-\frac{b}{a} x^{2} & -\frac{2 d}{a} x+\frac{2 e}{a} x^{2}
\end{array}\right] \\
& \tilde{\mathcal{E}}_{\mathrm{B}}^{K}=\left[\begin{array}{ll}
y-\frac{c}{a} x & -\frac{d}{2 a} y+\frac{f b}{2 a^{2}} x^{2} \\
-\frac{d}{2 a} y+\frac{f b}{2 a^{2}} x^{2} & \frac{f d}{a^{2}} x-\frac{2 e^{2}}{a^{2}} x^{2}
\end{array}\right] \\
& \tilde{\mathcal{E}}_{9}^{K}=\left[\begin{array}{ll}
0 & -\frac{c}{2 f} y+\frac{b}{2 f} y^{2} \\
-\frac{c}{2 f} y+\frac{b}{2 f} y^{2} & y-\frac{e}{f} y^{2}
\end{array}\right] \\
& \dot{\mathcal{E}}_{10}^{K}=\left[\begin{array}{ll}
-\frac{2 c}{f} y+\frac{2 b}{f} y^{2} & y-\frac{e}{f} y^{2} \\
y-\frac{e}{f} y^{2} & 0
\end{array}\right] \\
& \varepsilon_{11}^{K}=\left[\begin{array}{ll}
x y-\frac{c}{2 a} x^{2}-\frac{b}{2 a} x^{2} y & \frac{f}{4 a} x^{2}-\frac{d}{2 a} x y+\frac{e}{4 a} x^{2} y \\
\frac{f}{4 a} x^{2}-\frac{d}{2 a} x y+\frac{e}{4 a} x^{2} y & 0
\end{array}\right] \\
& \dot{\mathcal{E}}_{12}^{K}=\left[\begin{array}{ll}
y^{2}-\frac{2 c}{a} x y+\frac{b}{a} x y^{2} & \frac{f}{a} x y-\frac{d}{2 a} y^{2}+\frac{e}{2 a} x y^{2} \\
\frac{f}{a} x y-\frac{d}{2 a} y^{2}+\frac{e}{2 a} x y^{2} & 0
\end{array}\right] \\
& \overline{\mathcal{E}}_{13}^{K}=\left[\begin{array}{ll}
\frac{2 a}{f} x y^{2}-\frac{2 c}{f} x^{2} y & x^{2} y-\frac{d}{J} x y^{2} \\
x^{2} y-\frac{d}{f} x y^{2} & 0
\end{array}\right] \\
& \overline{\mathcal{E}}_{14}^{K}=\left[\begin{array}{ll}
0 & \frac{a}{4 J} y^{2}-\frac{c}{2 f} x y+\frac{b}{4 J} x y^{2} \\
\frac{a}{4 f} y^{2}-\frac{c}{2 f} x y+\frac{b}{4 f} x y^{2} & x y-\frac{d}{2 J} y^{2}-\frac{e}{2 f} x y^{2}
\end{array}\right] \\
& \overline{\mathcal{E}}_{15}^{K}=\left[\begin{array}{ll}
0 & \frac{a}{f} x y-\frac{c}{2 f} x^{2}-\frac{b}{2 f} x^{2} y \\
\frac{a}{f} x y-\frac{c}{2 f} x^{2}-\frac{b}{2 f} x^{2} y & x^{2}-\frac{2 d}{f} x y+\frac{e}{f} x^{2} y
\end{array}\right] \\
& \dot{\mathcal{E}}_{16}^{K}=\left[\begin{array}{ll}
0 & x y^{2}-\frac{c}{a} x^{2} y \\
x y^{2}-\frac{c}{a} x^{2} y & \frac{2 f}{a} x^{2} y-\frac{2 d}{a} x y^{2}
\end{array}\right]
\end{aligned}
$$




\section{Proof:}

Let $\mathcal{B}_{K}=\operatorname{grad} \mathcal{F}_{K}^{-1}$. Taking (10) into account we have in system $s O t$ :

$$
\mathcal{B}_{K}=\left[\begin{array}{ll}
a-b x & -d+e x \\
-c+b y & f-e y
\end{array}\right] J_{F}^{-1} .
$$

On the other hand, the gradient of every field $\mathrm{v}$ defined in $\Omega$ restricted to $K \in \mathcal{T}_{h}$ is given by:

$$
\operatorname{grad} v_{\mid K} \circ \mathcal{F}_{K}=\widehat{\operatorname{grad}} \hat{v} \mathcal{B}_{K}
$$

where $\hat{v}=v_{\mid K} \circ \mathcal{F}_{K}$ and $\widehat{\text { grad }}$ denotes the gradient in the reference plane $x \hat{O} y$. Hence by definition we have

$$
\mathcal{E}(v)_{\mid K} \circ \mathcal{F}_{K}=\left[\widehat{\operatorname{grad}} \hat{v} \mathcal{B}_{K}+\mathcal{B}_{K}^{T}(\widehat{\operatorname{grad}} \hat{v})^{T}\right] / 2
$$

In the particular case where $v \in V_{h}$, the space of each component of $\hat{v}$ is the one spanned by the monomials $1, x, y, x y, x^{2}, y^{2}, x^{2} y, x y^{2}, x^{2} y^{2}$.

Using this fact to expand grad $\hat{v}$, together with (14), the Lemma becomes the consequence of a straightforward calculation. q. e. d.

Now we derive a basis $\left\{\widehat{\mathcal{E}}_{i}^{K}\right\}_{i=1}^{M}$ of the space spanned by $\left\{\tilde{\mathcal{E}}_{i}^{K}\right\}_{i=1}^{16}$ in such a way that $\mathcal{E}(v)_{\mid K} \circ \mathcal{F}_{K}$ is alternatively expressed by (4). We establish in Lemma 2 below that actually $\mathrm{M}<16$.

Lemma $2 \forall v \in V_{h}$ and $\forall K \in \mathcal{T}_{h}, \mathcal{E}(v) / K \circ \mathcal{F}_{k}$ is given by (4) with $M=15$ where the $\widehat{\mathcal{E}}_{i}^{K}$ 's are given by

$$
\widehat{\mathcal{E}}_{1}^{K}=\tilde{\mathcal{E}}_{1}^{K}, \widehat{\mathcal{E}}_{2}^{K}=\tilde{\mathcal{E}}_{2}^{K}+\tilde{\mathcal{E}}_{3}^{K}, \widehat{\mathcal{E}}_{i}^{K}=\tilde{\mathcal{E}}_{i+1}^{K} \text { for } i=\overline{3,15}
$$

Proof:

Let us first establish that there are at most fifteen linearly independent tensors in the set $\left\{\tilde{\varepsilon}_{i}^{K}\right\}_{i=1}^{16}$. Indeed assume that

$$
\sum_{i=1}^{16} \gamma_{i} \tilde{\mathcal{E}}_{i}^{K} \equiv 0
$$

Through a trivial though lengthy identification process, we find out that identity (16) is satisfied if and only if the following relations hold, whereby we have set $\gamma_{7}^{\prime}=\gamma_{7}-\frac{f \gamma_{8}}{2 a}$ and $\gamma_{10}^{\prime}=\gamma_{10}-\frac{a \gamma_{6}}{2 f}$,

$$
\begin{array}{lll}
\gamma_{1}=\frac{c}{f} \gamma_{3} & \gamma_{2}=-\gamma_{3} & \gamma_{4}=\frac{d}{a} \gamma_{2} \\
\gamma_{5}=\frac{c^{2}}{a f} \gamma_{10}^{\prime} & \gamma_{6}=\frac{2 d}{a} \gamma_{7}^{\prime}-\frac{e}{a} \gamma_{2} & \gamma_{7}^{\prime}=\frac{-c}{a} \gamma_{10}^{\prime}=\frac{-f}{d} \gamma_{10}^{\prime} \\
\gamma_{8}=\frac{2 c}{f} \gamma_{10}^{\prime}-\frac{b}{f} \gamma_{3} & \gamma_{9}=\frac{d^{2}}{a f} \gamma_{7}^{\prime} & \gamma_{11}=\frac{-2 b c}{a f} \gamma_{10}^{\prime} \\
\gamma_{12}=\frac{-2 b}{f} \gamma_{10}^{\prime} & \gamma_{13}=\frac{-b f}{2 a^{2}} \gamma_{12} & \gamma_{14}=\frac{-2 e d}{a f} \gamma_{7}^{\prime} \\
\gamma_{15}=\frac{-2 e}{a} \gamma_{7}^{\prime} & \gamma_{16}=\frac{-e a}{2 f^{2}} \gamma_{15} &
\end{array}
$$


It is readily seen that if $\mathrm{cd} \neq$ af we have $\gamma_{7}^{\prime}=\gamma_{10}^{\prime}=0$, which implies that $\gamma_{5}=\gamma_{9}=\gamma_{11}=\gamma_{12}=\gamma_{13}=\gamma_{14}=\gamma_{15}=\gamma_{16}=0$. On the other hand, any vector $\vec{\gamma} \in \mathcal{R}^{16}$ of coefficients which is a multiple of $\left(\frac{c}{f},-1,1,-\frac{d}{a}, 0, \frac{e}{a}, 0,-\frac{b}{f}, 0\right.$, $0,0,0,0,0,0,0)$ satisfies identity (16) and conversely.

Summarizing, if $\mathrm{cd} \neq$ af we have $\mathrm{M}=15$, which means that one of the $\tilde{\epsilon}_{i}^{K}$ 's is linearly independent of the remaining fifteen. Notice that the relation $\mathrm{cd}=$ af implies that $\left(y_{4}-y_{2}\right)\left(x_{3}-x_{1}\right)+\left(y_{3}-y_{1}\right)\left(x_{2}-x_{4}\right)=0$. Since this situation is discarded by a simple geometric argument (the vector product of both diagonals of $K$ must not vanish), we effectively have cd $\neq$ af.

On the other hand, as one may easily verify $\sum_{i=1, i \neq 3}^{16} \gamma_{i} \bar{e}_{i}^{K} \equiv 0$ implies that $\gamma_{i}=0 \quad \forall i, i \neq 3$.

Hence the space spanned by $\left\{\tilde{\varepsilon}_{i}^{K}\right\}_{i=1}^{16}$ is the same as the one spanned by $\left\{\tilde{\mathcal{E}}_{i}^{K}\right\}_{i=1, i \neq 3}^{16}$, or yet by the set $\left\{\overline{\mathcal{E}}_{i}^{K}\right\}_{i=1}^{15}$ defined in (15) q. e. d.

Let us now set

$$
r_{K}^{\prime}=\frac{a}{f}=\frac{2 r_{K}-r_{K} \alpha^{2}\left(\beta_{1}^{2}+\beta_{2}^{2}\right)+\alpha^{3}\left(\beta_{2}^{2}-\beta_{1}^{2}\right)}{2+r_{K} \alpha\left(\beta_{2}^{2}-\beta_{1}^{2}\right)-\alpha^{2}\left(\beta_{1}^{2}+\beta_{2}^{2}\right)}
$$

and make the following assumption:

Assumption A: There exists two strictly positive constants $\rho$ ' and $\xi$ ' independent of K such that $r_{K}^{\prime}$ is bounded above and below by $\frac{1}{\xi}$ and $\rho$ respectively.

Notice that Assumption A holds if we have

Assumption B: $\quad \delta_{K}<\min \left[2^{-1 / 4},\left(2 r_{K}\right)^{-1 / 3}\right]$

Indeed in so doing we have

$$
\frac{r_{K}}{1-2 r_{K} \delta_{K}^{3}} \geq r_{K}^{\prime} \geq \frac{r_{K}\left(1-2 \delta_{K}^{4}\right)}{1+2 r_{K} \delta_{K}^{3}}
$$

Thus we may take

$$
\xi^{\prime}=\inf _{K \in \mathcal{T}_{h}} \frac{1-2 r_{K} \delta_{K}^{3}}{r_{K}}
$$

and

$$
\rho^{\prime}=\inf _{K \in \mathcal{T}_{h}} \frac{r_{K}\left(1-2 \delta_{K}^{4}\right)}{1+2 r_{K} \delta_{K}^{3}}
$$

Henceforth we make Assumption B on mesh $T_{h}$.

Notice that one of the consequences of Assumption $B$ is the fact that the angles $\theta,\left|\phi_{1}\right|$ and $\left|\phi_{2}\right|$ are smaller than $\pi / 4$.

Now we have: 
Lemma 3 The set $\left\{\widehat{\mathcal{E}}_{i}^{K}\right\}_{i=1}^{15}$ given by (15) for which (4) holds, may be written as:

$$
\widehat{\mathcal{E}}_{i}^{K}=\widehat{\mathcal{E}}_{i}^{K}+\Delta_{i}^{K} \quad i=\overline{1,15}
$$

where $\widehat{\mathcal{E}}_{i}^{K}$ is given by the same expression as $\widehat{\mathcal{E}}_{i}^{K}$ in (7), if one replaces $r_{K}$ with $r_{K}^{\prime}$, and where the $\triangle_{i}^{K}$ 's are tensors whose $L^{2}(\widehat{K})$-norms are all bounded above by a term $C \delta_{K}$, where $C$ is a constant independent of $K$.

\section{Proof:}

This result is a trivial consequence of (13), (15) e (12). q. e. d.

Now just for convenience we will work with a local basis $\left\{\widehat{\sigma}_{j}^{K}\right\}_{j=1}^{15}$ defined as a suitable linear combination of the tensors $\sigma_{j}, \mathrm{j}=\overline{1,15}$ with coefficients independent of $\mathrm{h}$, whose main property is given in

Lemma 4 For every $K \in \mathcal{T}_{k}$ there exist fifteen linearly independent tensors $\left\{\widehat{\sigma}_{j}^{K}\right\}_{j=1}^{15}$ belonging to the space spanned by $\left\{\sigma_{j}\right\}_{j=1}^{15}$ given by $(6)-\left(6^{\prime}\right)$ that satisfy

$$
\int_{\widehat{K}} \widehat{\mathcal{E}}^{\prime}{ }_{i}^{K} \cdot \widehat{\sigma}_{j}^{K} d \widehat{x} d \widehat{y}=\delta_{i j}
$$

Proof: According to [RUA 93] if $\mathrm{K}$ is a rectangle, matrix $A=\left\{a_{i j}\right\}$ given by (5) has the following properties:

First of all, the diagonal terms of $\mathrm{A}$ are the following strictly positive numbers independent of rectangle $\mathrm{K}$ :

$$
\begin{gathered}
a_{j j}=\frac{16}{9} j=1,2 ; \quad a_{33}=\frac{32}{9} ; \quad a_{j j}=\frac{16}{45} j=4,5,7,8 ; \\
a_{j j}=\frac{32}{45} j=6,9 ; a_{j j}=\frac{32}{225} j=10,13 ; \quad a_{j j}=\frac{128}{1575} j=11,14 ; \\
a_{j j}=\frac{32}{525} j=12,15
\end{gathered}
$$

On the other hand the only non zero off-diagonal terms of matrix $B=\left\{b_{i j}=\right.$ $\left.(\operatorname{diag} A)^{-1} A\right\}$, are :

$$
\begin{aligned}
& b_{11,1}=b_{14,2}=b_{12,9}=b_{15,6}=\frac{1}{5} ; \quad b_{9,12}=b_{6,15}=\frac{7}{3} ; \\
& b_{10,3}=\frac{1}{10 \mathrm{r}_{\mathrm{K}}} ; b_{13,3}=\frac{\mathrm{r}_{\mathrm{K}}}{10} ; \quad b_{12,4}=\frac{2 \mathrm{r}_{\mathrm{K}}}{5} ; \quad b_{15,8}=\frac{2}{5 \mathrm{r}_{\mathrm{K}}}
\end{aligned}
$$

Now set $\sigma_{j}^{\prime}=\sigma_{j} / a_{j j} j=\overline{1,15}$. By choosing 


$$
\begin{gathered}
\widehat{\sigma}_{12}^{K}=\left(15 \sigma_{12}^{\prime}-35 \sigma_{9}^{\prime}\right) / 8 ; \widehat{\sigma}_{15}^{K}=\left(15 \sigma_{15}^{\prime}-35 \sigma_{6}^{\prime}\right) / 8 ; \\
\widehat{\sigma}_{1}^{K}=\sigma_{1}^{\prime}-\frac{\sigma_{11}^{\prime}}{5} ; \widehat{\sigma}_{2}^{K}=\sigma_{2}^{\prime}-\frac{\sigma_{14}^{\prime}}{5} ; \widehat{\sigma}_{3}^{K}=\sigma_{3}^{\prime}-\frac{\sigma_{10}^{\prime}}{10 r_{K}}-\frac{r_{K} \sigma_{13}^{\prime}}{10} \\
\widehat{\sigma}_{4}^{K}=\sigma_{4}^{\prime}-\frac{2 r_{K} \hat{\sigma}_{12}^{K}}{5} ; \quad \widehat{\sigma}_{6}^{K}=\sigma_{6}^{\prime}-\frac{\widehat{\sigma}_{15}^{K}}{5} ; \quad \widehat{\sigma}_{8}^{K}=\sigma_{8}^{\prime}-\frac{2 \widehat{\sigma}_{15}^{K}}{5 r_{K}} ; \\
\hat{\sigma}_{9}^{K}=\sigma_{9}^{\prime}-\frac{\widehat{\sigma}_{12}^{K}}{5} ; \text { and } \hat{\sigma}_{j}^{K}=\sigma_{j}^{\prime} \text { for } j=5,7,10,11,13,14
\end{gathered}
$$

a straightforward calculation leads to:

$$
\int_{\widehat{K}} \widehat{\mathcal{E}}_{i}^{K} \cdot \widehat{\sigma}_{j}^{K} d \widehat{x} d \widehat{y}=\delta_{i j}
$$

where the $\widehat{\mathcal{E}}_{i}^{K}$ s are associated with rectangle $K$ (see $(7)$ ).

The Lemma is then a simple consequence of the definition of the ${\hat{\mathcal{E}}^{\prime}}_{i}^{K} s$ from the $\widehat{\mathcal{E}}_{i}^{K}{ }^{*}$ given by (7), as specified in Lemma 3. q. e. d.

It follows from Lemma 4 that, matrix $A_{K}=\left\{\left(a_{K}\right)_{i j}\right\}$ defined in the same manner as $A=\left\{a_{i j}\right\}$ in (5), by replacing $\sigma_{j}$ with $\widehat{\sigma}_{j}^{K}$ is of the form

$$
A_{K}=I+\Delta A_{K}
$$

where

$$
\Delta A_{K}=\left\{\left(\Delta a_{K}\right)_{i j}\right\}
$$

with

$$
\left(\Delta a_{K}\right)_{i j}=\int_{\widehat{K}} \Delta_{i}^{K} \cdot \widehat{\sigma}_{j}^{K} d \widehat{x} d \widehat{y} .
$$

As a final preparatory result we have

Lemma 5 If parameter $\delta$ given by (8) is sufficient small matrix $A_{K}$ is invertible $\forall K \in \mathcal{T}_{h}$. Moreover there exists a constant $C$ independent of $h$ such that

$$
\left\|A_{K}^{-1}\right\| \leq C \quad \forall K \in \mathcal{T}_{h} \text { and } \forall \mathcal{T}_{h} \text { (18). }
$$

According to Assumption B which implies upper and lower bounds for $\boldsymbol{r}_{\boldsymbol{k}}^{\prime}$ given by (17), and recalling (16), (13) and (12), the moduli of all the coefficients of matrix $\Delta A_{K}$ are bounded above by a term of the form $\mathrm{c} \delta_{K}$, where $\mathrm{c}$ is a constant independent of $\mathrm{K}$. Therefore, from well-known results, matrix $A_{K}$ will be invertible, provided $\delta_{K}$ is small enough to yield $\left\|\Delta A_{K}\right\|<1$, where $\|$. represents any consistent matrix norm. 
Moreover, if parameter $\delta$ is sufficiently small then there exists a constant $\gamma$ $<1$ such that $\left\|\Delta A_{K}\right\|<\gamma \quad \forall K \in \mathcal{T}_{h}$ and $\forall \mathcal{T}_{h}$. Therefore we have

$$
\left\|A_{K}^{-1}\right\| \leq \frac{1}{1-\left\|A_{K}\right\|} \leq \frac{1}{1-\gamma} \quad \forall K \in \mathcal{T}_{h} \text { and } \forall \mathcal{T}_{h} .
$$

which proves the Lemme. q. e. d.

As pointed out in Section 2, Lemma 5 is sufficient to establish (3). As a consequence, recalling again the arguments given in [FOR 88], we have the following convergence result:

Theorem 1 If the solution $(\sigma, u, p)$ of system (1) as such that $u \in H^{3}(\Omega)$, and $p \in H^{2}(\Omega)$, approximate solution $\left(\sigma_{h}, u_{h}, p_{h}\right)$ of system (2) satisfies:

$$
\left|u-u_{h}\right|_{1}+\left\|\sigma_{h}-\sigma\right\|_{0}+\left\|p-p_{h}\right\|_{0} \leq \mathcal{C}(u, p) h^{2}
$$

where $\mathcal{C}$ is a constant independent of $h$, provided the quasiuniform family of partitions $\left\{\mathcal{T}_{h}\right\}_{h}$ is such that the distortion parameter $\delta$ given by $(8)$ is not too large.

\section{Concluding Remarks}

i) The same results proved in this paper should apply to the element considered in [RUA 91] in which $T_{h}^{2}$ is a space with local dimension equal to twelve instead of fifteen. However, not only the corresponding analysis is technically more complex, but this element only works if the pressure space $Q_{h}$ consists of discontinuous piecewise linear functions (for the same $V_{h}$ as here!).

ii) The boundedness of the three angular increments used in this paper as a distortion measure of quadrilaterals seems to be only a formal requirement. Indeed, in all the computer tests for the Stokes system performed so far with arbitrary quadrilaterals, the new finite element method showed both stable and accurate behaviour, irrespective of the distortion degree of the mesh. These results will be included in a forthcoming paper on viscoelastic flow simulations. ii) $T_{h}^{2}$ is defined as a space spanned at the level of a quadrilateral $K$ of the mesh, by $\left\{\sigma_{K}^{j}\right\}_{j=1}^{15}$, where $\sigma_{j}^{K}=\sigma_{j} \circ \mathcal{F}_{K}^{-1}$, with $\sigma_{j}$ given by (6)-(6'). Therefore $T_{h}^{2}$ is related to the frame which mappings $\mathcal{F}_{K}$ are referred to. Here this means dependence on the local basis $(\vec{s}, \vec{t})$, which in turn depends on $K$. Thus, computation with such $\sigma_{K}^{j}$ requires in principle a post-processing of the mesh, in order to determine the axes $O \vec{s}$ and $O \vec{t}$ for each element, together with corresponding rotation matrices with respect to a fixed cartesian frame. Strictly 
speaking, the latter are to be applied to $\sigma_{j}$ for $j=\overline{10,15}$, in each element. Notice however that in the context of viscoelastic flow simulations, such additional computational effort is negligible.

iv) Finally it should be noted that the finite element method studied in this paper has the same convergence properties as the one introduced by MARCHAL \& CROCHET in [5]. Notice that for both elements the velocity representation is the same, together with the one of the pressure, as long as it is constructed upon continuous $Q_{1}$ (isoparametric bilinear) functions. The essencial difference lies in spaces $T_{h}$ defined in this work and in theirs. Let us recall that in the latter, $T_{h}$ consists of continuous $Q_{1}$ functions based on a quadrilateral mesh four times finer than $\mathcal{T}_{h}$.

Although the use of space $Q_{\perp}$ could show some computational advantages, the author would like to emphasize that in this approach the number of extra stress degrees of freedom increases roughly by a factor of three, compared to the present one, when related to the same velocity mesh $\mathcal{T}_{h}$. In any case this author feels that it would be wise to perform extensive experiments with both methods, in order to compare their performances in the framework of classical test problems in viscoelasticity.

\section{Acknowledgment}

This paper was prepared in July/August 1991 at the Department of Computer Science of the Catholic University of Rio de Janeiro. The author would like to thank his doctoral students, J. H. CARNEIRO DE ARAUJO, M. A. M. SILVA RAMOS and P. R. TRALES for having so kindly contributed to the successful accomplishment of this research work.

\section{References}

[BER 79] M. BERCOVIER \& O.PIRONNEAU, "Error Estimates for the Finite Element Method Solution of the Stokes Problem in the Primitive Variables", Numer. Math., 33 (1979), 211-224.

[CAR 91] J. H. CARNEIRO DE ARAUJO, Métodos de Elementos Finitos Otimizados para - Sistema de Stokes Associado a Problemas de Viscoelasticidade, Doctoral dissertation, Pontifícia Universidade Católica do Rio de Janeiro, 1991.

[FOR 89] A. FORTIN \& M. FORTIN, "A New Approach for the Finite Element Simulation of Viscoelastic Fluws", Jour. Non-Newtonian Fluid Mech., 32 (1989), 295-310. 
[FOR 88] M. FORTIN \& R. PIERRE, "On the Convergence of the Mixed Method of Crochet \& Marchal for Viscoelastic Flows", Comp. Meth. Appl. Mech. Engin., 73 (1988), 341-350.

[MAR 87] J. M. MARCHAL \& M. CROCHET, "A New Finite Element for Calculating Viscoelastic Flow", Jour. Non-Newtonian Fluid Mech., 26 (1987), 77-114.

[RUA 85] V. RUAS, "Une méthode mixte contrainte-déplacement-pression pour la résolution de problèmes de viscoélasticité incompressible en déformations planes", Comptes Rendus de l'Académie des Sciences de Paris, t. 301, Série II, 16 (1985), 1171-1174.

[RUA X] _ _ _ _ , An Optimal Second Order Three Field Finite Element Method for the Stokes System with Continuous Extra Stresses", to appear.

[RUA 91] V. RUAS, J.H. CARNEIRO DE ARAUJO \& M.A.M. SILVA RAMOS, "Finite Element Models for Viscuelstic Liquids: Some Recent Developments", in: Numerical Methuds in Laminar and Turbulent Flow, Vol VII, Eds. C. Taylor, J.H. Chin, G.M. Homsy, Pineridge Press, Swansea, 1991, 1658 - 1668.

[RUA 93] _-___ " Approximation of the Three-Field Stokes System Via Optimized Quadrilateral Finite Elements", to appear in RAIRO - Modélisation Mathématique et Analyse Numérique.

[RUA 92] V. RUAS \& J. H. CARNEIRO DE ARAUJO, "Un método mejorado de segundo orden para la simulación de flujo viscoelástico con elementos finitos cuadrilaterales", Revista Internacional de Métodos Numéricos para cálculo y Diseño en Ingeniería, vol. 8, no 1, (1992), $77-85$.

[SAN 91] D. SANDRI, Analyse Numérique de Fluides Non Newtoniens: Fluides Viscoélastiques et Fluides Quasi-Newtoniens ,Thése de Doctorat, Universite Claude Bernard - Lyon I, Lyon, 1991.

\section{Curriculum Vitae}

Docteur d'Etat (1982) in Numerical Analysis (NA), Université Paris VI. Professeur of NA at PUC/RJ since 1982. Professeur de Mécanique a l'Université de Saint Etienne since 1989. Member of Laboratoire de Modélisation en Mécanique, UPMC/CNRS, Paris, since 1988. 